\title{
VII. \\ Die Entwicklungslinie der Philosophie im Kulturbereiche des Islam.
}

Eine Skizes von

Dr. M. Horten. Bonn.

Fïne jede gristige Betätigung ist notgedrungen bestimmt durch die umgebende Kiultur. Die im Kulturbereiche des Islam entstandene Philosophie entnimmt dementsprechend wesentliche Momente aus der Wolt- und Lebensanschaumng des Is a m und differenziert sich dadurch von der Philosophic, die in den beiden angremzenden Kultur-

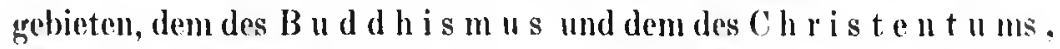
aufgetreten ist. Zu diesen Einflïssen allgemeiner Natur gesellen sjch speziell philosophische, die ihre Quelle in den anliegenden Ländern, Indien und Griechenland, haben.

Sobald sich die stürmischen Fluten der arabischen Völlierwanderung geebnet baben (gegen 750), entfaltet sich unter diesen Bedingungenl ein eigentümliches, sehr reges geistiges Ichen, das scine Hauptsitze in Iran, Mesopotaunien, Syrien, $\ddot{\text { ggypten }}$ und Spanien hat (also in den Ländern, de im Besitze alter Kulturgïter standen, deren Fibre dor Islam antrat). Den Kulminationspunkt seines Schaffens erreicht es im XI. his XV. Jahrhundert. Seitdem danert es unter beständigen Schwankungen bis in unsere Tage, also zwölfhundert Jalıre hindured, fort, um seit ungefähr 1850 unter dem erweckenden Hauche der modemen europäischen Kultur einen neuen $\Lambda$ ulschwung zu nebmen. Es sind vier Hauptrichtungen, in denen sich die philosophischen Bestrebungen dieser Geisteskultur auswirken, die griechische, dir innerislamische (theologische), die geistes- und naturwissenschaftliche (nichttheologische), die mystische.

1. Die griechische Jonkrichtung ist bestrebt, die griechische Philosophie dem Islam zu vermitteln. Dic Vorarbeiten zu diesem Ziele sind die Ubersetzungen der griechischen Philosophen, rine Aufgabe, der vor allem die Syrer gewachsen waren, da bei ihnen die Schätze 
griechischen Denkens weiterlebten und sie anch des Arabischen, der Sprache der damaligen Verkehrswelt und der Beherrscher Syriens, kundig waren. Durch Kosta bn Luca um 835, Honain bn Ishar, $873 \dagger$ Ishay bu Honain 911† und andere wurden in 1X. Jahrhundert die Werke des Aristoteles und seiner Kommentatoren Alexandor von Aphrodisias und Themistius, dic Platos und Auszïge aus den Emneaden IV-VI Plotins ins Arabische übersetz.t. Das letzte Werk hat insofern ein eigentümliches Geschick erlebt, als es unter dem Namen der Theologie des Aristoteles in Lmlauf kam. Das Bild, das sich die orientalische Philosophie von distoteles machte, wurde daher stark mit plotinischen Zügen ausgestattet, und die arabischen l'hilosophen hiclten sich für Schüler des Aristoteles, während sie ebensogut Schüler Plotins sind. Den gleichen Einflub hatte die psendo-aristotelische Schrift „tber das reine Gute", auch liber de causis genannt. Sie ist ein Exzerpt aus

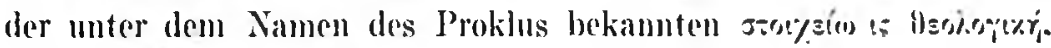
d. h. der Elomente der Metaphysik.

Auf diese Voraheiten baut sich nun die griechisch gefäbto benkrichtung auf, die der Falásifa ${ }^{1}$ ) (der Akzent bezeichnet die Stelle des arabischen 'Tones). Sie allein sind in den europäischen Ländern als „Pliilosophen“ bekamnt geworden, weshalb die einfache Auf\%ählung genügen möge: Kindi (Alkíndi) um 870, Farábi (Alfarabius) 950†, ibn Śmar (Avicenna) 1037 - er vermittelte dem Jslam das Verständnis der griechischen Philosophie - ibn Róschd (Averróes) .1198†. Letzterer lant hauptsächlich durch seine Kommentare zu Aristoteles (Lohre von der Einheit des aktiven und passiven Intellektes) anf die Scholastik eingewirkt, ist aber für die Entwicklung der Philosophie innerhalb des Islam olune bedeutung geblicben. Dasselbe gilt von ibu Bággai (Badscha, Avempace) $1138+$ und ibu 'lufail 118j) f. Das System dieser setzt sich aus droi Komponenten zusammen: aristotolischr, neuphatonisehe und koranische Jdeen. Mhr Verhälnis ist so, dab dio aristotelische Lleongruppe (Logik, liosmologie, Metaphysik) die Bausteine liefert, die neuplatonische Ideemgruppe ('Theologie, Limaniltionslehre, Psychologie) das Ganze architektonisch ordnet und ilm

") Dieses griechische Jremolwort wurde fiilschlich mit ,. Philosophen" übor-

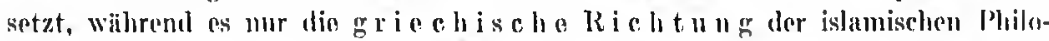
sophen bezeichmet. Nlo Nicht-Falasifa galten daher als Nicht-Philosophen. und mit dem Aufhören der Falisifa lieb man auch in den I)arstellumgen der , ,arabisclien Philosophie" diese sellsst zu Finde gehen. 
ein clarakteristisches Gepräge gibt. Die koranischen Vorstellungen haften dem (iedankenbau nur äußerlich an. D)as System stellt sich also als einen neuplatonischen Aristotelismus dar. Im einzelnen ist noch zu bemerken, dab die ,Plilosophen" Gott als Intellekt auffassen. Lir erkennt sich und die Geisterwelt direkt, und in den sekmdären Ursachen anch die matericlle Welt, und zwar nicht nur die Allgemeinbegrifie der Jinge, sondern anch die Individua. „Kein Atom in Himmel und auf Erden entgeht lhm." Ciott schafit er k e 11 n e $n$ d. Die Wolt ist o w i g e Emanation Gottes, a 11 f a $n \mathrm{gs}$ los, da Cottes Denken ohne Anfang und notwendig ist. Wie Denk-

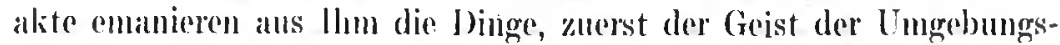
sphäre, aus diesem unter dem Mitwirken Gottes die Gister, Seclen und liörper der acht äbrigen Sphären. Der Geist der Mondsphäre ist derjenige, der als aktiver Intellekt dio Potentialitat unseres Denkvermögens in die Tätigkeit, das akturelle IJenken, überfïhnt. Wenn wil also die Iresenheiten aus den Dingen abstrahieren, so emplangen wir diese Aktivität von dem aktiven Intellekte - und mit der Aktivitait anch den Jenkinhalt. Jenn ebenso wie die wesenlose Materie der matericllen Dinge von dem Geiste der Mondsphäre ihre realen Wesenheiten erhält, so ertält anch unser an sich ideenloser, potentieller, passiver Geist von diesem aktiven Intelledite die logischen Wesenheiten, d. h. seine Begrille mal Erkemntnisse. Als Sit\% Ciottes ist, weil ruhender Punkt des Woltalls, der Nordpol des Jimmols gedacht. Die Vollkommenheit hesteht in dem stroben slach Gott und dom Abstreifen der Materie. In den geschöpflichen Dingen ist das Dascin mur zufällig mit den Wesenheiten verbunden. Aus sieh heraus besitzen die Dinge also kein Dasoin. Sie müssen es daher von der Cottheit, der Quelle des Seins, die das Scin als Wesenheit besitzt, ('m)fiangen (hontingenzheweis). Jodes neu auftretende Wirkliche muB cone adäguate, notwendig wirkende Vysache haben. Jeder erste Willensentschluß ist nun aber ein solches Wirkliche. Folghich mubs or eine aläquate Ursache haben, die in den Naturkräften und den Einwipkungen der Sterne zu suchen ist. Wer Wille des Mensehen ist also durch die Naturkäafte de terminiert, er ist abor l rei von einem direkten Ëinflusse Gottes. Dieser erstreckt sich nur auf den Cieist der Umgebungssphäre. Eine populäre Darstellung platonisch-pythagoräischer Philosophie entwarfen im 10. Jahrhundert die Getreuen von Basra, fälschlich die lauteren Brïder genamnt. 
11. Die inner-islamische theologische Denkrichtung (die der Mutakallimún) stellen die ersten Versuche dar, das koranische Dogmensystem mit der Vernunft zu veroinigen. Diese Bestrebungen nehmen sich ans wir die ersten tastenden Gehrersuche oines Kindes, sind aber als Anfangsphase ciner philosophischen Bewegung historisch und psychologisch überaus interessant. Das Dogma besitzt zunächst die Vorherrschaft ühor die Vermunft bis Wásil bu Atá 748 f die Schule der ,"Wreidenlier" (Mútazila, Mutaziliten) gründete, die bald (gegen $8(0)$ griechischeol ldeon Einlab gewährten -- allzuviel für das Empfinden islamischer Orthodoxie. So mubte cine Reaktion gugunsten des Dogmas cintreten. Aschari 873-935 ron Basra suchte die beiden streitenden Parteien zu grö̈Berer beiderseitiger \%ufriedenheit zu vereinigen. ber Giucksgriff ist jedoch anch ihm und seiner Schule nicht gelungen, wie (iaźli zeigt. Die hier genamnten Schulen haben

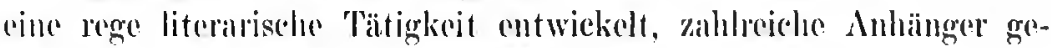
habt und bis in die \%oit mach ( vakali bestanden. Ihre schicksale sind im einzchen noch nicht aufgehellt. Die Diskussionen erstrecken sich anf den Gottesbegrifi, EünfuB Gottes anf die Freiheit des IVillens, das Böse und das (ieschehen in der Natur. Die Atomtheorie worde angenommen mud das Wirken der Naturkrafte zugunsten des Wirkens Gottes von der orthodoxen Richtung aufgehoben.

III. Die natumissenschaftliche mol geisteswissenschaftliche Richtung ist als eine phitosophisehe zu bezeichnon, weil zumähst die Xaturwissenschalten als 'Tril der l'hilosophic gerechnet wurden, sodamm in ihmen manche philosophisehen Themata behandelt werden (liosmologie, Psychologic usw.) und vielfach die Naturwissenschaftler, bekannt mit allgemein spekulativen Untersuchumgen, zu den phi-

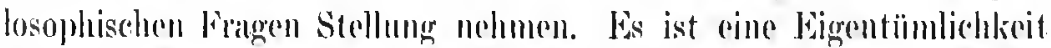
der damaligen Líntur, dab jeder Gobildete P'labosoph sein mubte. biese Richtung schö̈pfte naturgemäß reichlich aus griechischen Quellen, wird abor nicht als cine griechische empfumben, da noben den griechischen Quellen ihr anch indische rejehlich zuflossen umd ilure Vertroter den Quellen gegenïber den Wert sellsstandiger Forscher heanspruchen. Bekannt sind ror viden anderen 'lábit bu kora 901 f (\%ahlentheorio), Fargáni um 830 (Alfarganus) und Battáni 929 (. llhategnius, Astronomen und Astrologenl), Rázi 932 (Rases, Arat), (ráhir bu Hajản $1 \mathrm{~m} 760$ (Alchimist). Ihn al - Haitam 1038 † erkannte durch scharfsimnige Analyse bereits die apperzeptiven Elemente der 
Wahrnehmung; dem diese setzt sich nach ihm aus dem gegenwärtigen Empfindungsinhalte und dem Erinnerungsbilde früherer Empfindungen zusammen. Viel genannt sind der Dichterphilosoph und Pessimist. Abulalá al-Maárri $1057+$ und der Atheist Omar al Haijám 1121 $\%$. Besonders sind hier die Enzyklopädisten zu nemnen, die die Gesamtleit der Einzelwissenschaften zu einem einheitlichen Bilde zusammenfassen wollen, eine rein philosophische Autgahe: Gauzi $1200+$, der Gegner Gazális, Rári (Fahraddin) 1.209 t, ein extremer Realist in philosophischen Begriffen und sein Kommentator und Antipode 'Túsi $1273 \mathrm{t}$, der in erkenntnistheoretischen Fragen einen kritisch gemäBigten Realismus vertritt.

IV. Die mystische Denkrichtung ist nicht Jur (ino speliulative Weltanschauung, sondern auch eine praktische Lebensrichtung; sio ist auch $\Lambda$ skese. In Syrien schliebt sich dieselbe an christliche Vorbilder an, in Iran an buddhistische. Die Mystiker, Súlis genamnt, leben ein der Welt abgewandtes Jeben, halten sich dabei aber violfach nicht strenge an die Vorschriften des Islam. Sie sind erfüllt von neuplatonischen Ideen, die sie mit indischem Pantheismus weiter ansbanen: Gott ist die Fülle des Seins. Aus ihm entsteht durch innergöttliche Diflerenzierung die reale Welt. Deren Wesen ist also identisch mit Gott selbst. Auch die äubersten Konsecuenzen wurden daraus gezogen: der Mystiker darf Wein trinken, ohne daß or dadurch das Verbot des Propheten übertritt; denn das, was er trinlit, ist nicht Wein, sondern Gott selbst! Aus demselben Grunde ist allell die menschliche Seele cine Differenzierung des göttlichen Wesens und mulib daher beim Ableben des Körpers wiedermm in das unendlicho Heer des göttlichen Seins zurïreksinken (Nirvana), aus den sie wie dine kleine, flïchtige, spielende Welle aufgetaucht war.

Die Anfänge der islamischen Mystik liegen um 800. Sie tritt in riner dem Anachoretentum ähnlichen Form in $̈$ gypten und Syrien, als Klosterleben im Osten des islamischen linlturbereiches auf. wo die Einflüsse aus dem Baddhismus beständiger und lobendiger waren. Dic interessantesten Persönlichkeiten aus der fast unabsehbar grolien Zahl mystischer Philosophen sind: Hallág 921 t, der sich Gott (hatg = die Wahrheit, ein Name Gottes) nannte und für diese Bchauptung unter den unmenschlichsten Qualen sein Leben hingegeben hat, Koscháiri $1074+$ in Nisabur, Giláni 1166 t, der den Derwischorden der Qadirija stiftete, und Suhrawárdi, der die Wissenschaft von der 
göttlichen Frleuchtung aulstellte. 1191 starb er zu Neppo als Märtyrer sciner (Jberzelugung. In Murcia trat ibn al-Arabi als Mystiker auf. Er verbreitete scine Jehren in zahlreichen schrilten bis in dest fernen Osten. 1240 starb er in Damaskus. Kónawi $1273+$ aus Konia ist sein bedeutendster Schü̈ler.

Die genannten vier Richtungen bilden konvergierende linien, wie überhaupt in jeder Kultur dic verschiedenen Bestandteile sich gegenseitig beeinflussen und auszugleichen suchen. 1)ie Vertreter der Einzelwissenschaften auf den Gebieten der Satur und des Geistes sind unterrichtet über die anderen Richtungen. Diese hinwiederum Iernen von jenen. Die Philosophen sind auch Naturwissenschaftler (Avicema) und Mystiker (Faráhi), die Mystikor auch Philosophen (z. B. Faráni $\mathrm{mm}$ 1490). Die Theologie ist ferner die alles beherrschende und von allen gekannte Wissenschaft. Als wesentliche Bestimmung der islamisehen Geisteskultur besonders nach Gazili ist also ein gegenseitiges Jur(hdringen und Durchweben aller Richtungen zu konstatieren, d. h. exkl. Strömungen gibt es nicht. In dem breiten und homogenen Strome der Bildung finden sich jedoch insoforn Verschiedenheiten, als der eine sich auf diesem, der andere anf jenem Gebiete melr betätigt hat. Jeder Philosoph beherseht aber mehr oder weniger die ganze Fülle des zeitgenössischen Wissens. Fü̈r dic Technik der Darstellung ist es daher angeb)rach, daß für die Plilosophie seit dem 12. Jahrhundert die vier Richtungen nicht mehr als Eintoilungsprinzipien gelten.

In Mittelpunkte dieser Vereinigung aller Richtumgen steht Gazáli (siche oben) 1111 t. Jir ist Mystiker, Jheologe und Philosoph. Die damaligen Resultate der naturwissenschaftlichen lorschung nimmt or ohme Einschränkung an. Er hat dem Islam, wie Thomas von Apuin dem Christentume, die Tore der Wissenschaft geöffnet. Seit ihm nimmt die islamische Theologie wie soit 'Thomas die christliche rin griechisches Gewand an. J) Lederen der Mutukallimu befriedigten nicht seinen Geist, die der griechisch gerichtelen Denker nicht sein religiöses (iemiït. Beide Mängel hat ar ansgeglichen, ohne. unwissenschaftlich zu werden. Eine glückliche livitik übte er an $\mathrm{dem}$ Gotteshegrifle der griechischen Richtung. Cott kann keine reine Intelligenz sein, aus der sich nach Art logischer Deduktion die konkreten Weltdinge ergeben. Gott ist viedmehr als Wille, als Energie zu fassen, die freilich auch im löchsten Mabe intelligent ist. Der 
menschliche W:lle ist ferner nicht durch die Naturkräte notwendig determiniert (Lehre $\Lambda$ vicennas und Farábis), sondern wird direkt durch Gott in 'Tätigkeit versetzt (vgl. Thomas von Aquin). Die Ewigkeit der Welt ist philosophisch möglich, aber nicht erweisbar, noch Tatsache (ebenso Thomas). Das innere Wirken der Naturkräte kömnen wir nicht erschanen und dïrfen die Denkkategorie des hinreichenden Grundes nicht als transsubjektive Kausalität in die Dinge der AuBenwelt hineinlegen (vgl. Sextus empiricus, Hume und die Phänomenalisten). Diese lehore ist von den Mutakallimún übernommen. Garáli hat die Philosophice nicht vernichtet - sein cigenes System ist Philosophie -, sondern das gröBtmögliche Maß3 der Philosophic in die Theologie hinübergenommen. Iir ist im $\Lambda$ bendland durch seine "Vernichtung der Philosophen" hauptsächlich bekannt geworden und gill daher als Vornich ter der Philosophie. Es sind aber nicht die Philosophen im allgemeinen Simne, die er bekiimpft, sondern mur die Talásila, d. h. die griechische Richtungr der Philosophom. Dahori entwickelt or aus den Jehren seiner Gegner Konserquenzen, die diese n i c h t vertreten haben. Er läßst sie lehren, (iott erkemene nur die miversellen J)inge, nicht die Individua, und die Wolt sei unerschalfen. Im Cirunde ist die Schrift Gazális mur ein Streit 111 G G a $n$ \% f a ge $n$ zwischen Philosophie und Theologic. Garáli ist bestrebt, das Gobiet der letzteren auszudehnen, indem or mehrere 'Thesen, die die Philosophen als wissensehaftlich beweishar hinstellen, als mur durch (Offenbarung arkemmbar bezcichnet. Die folgende Entwicklumgr hat ihm aber darin Unrecht gegoben; demn nach Gazali ist eine noch bedeutend gröbere Anzahl von Philosophen aufgetreten und diese haben dieselben und anch noch wenigar orthodoxe Ansichten vertreten als vor ihm. lir hat weder die Philosophen noch auch nur die griechische Richtung der solben vernichtet, abensowenig wie 'Thomas ron Aguin dureh seine Bodämpfung der Averroisten, die sich, ,die wahren Aristoteliker" namiten, den Aristotelismuss int Abendlande vernichtet hat.

Im den Cberblick über das (iamze dentlich zu machen, lassen sich als Entwicklungsphasen der islamischen Philosophir vier, allerdings mit flichenden Grenzen, aufstellen:

1. Die Phase des Kampfes um tine philosophische Weltaulfassung boi der Anmahme griecluscher und indischer ldeen - 1050). 
2. Die Phase der Ausbildung der erstrebten Weltanschaumng, der Kritik und des Ausscheidens für den Islam nicht assimilierbarer ldeen (Gazáli) - 1150 .

3. I)ie Phase des ruhigen Besitzes, der Meiterbildung und des Ausbaues im lileinen - 1850).

4. Daran schließt sich dic letzte Phase an, die sich durch dio Aufnahme modern-europäischen Gristeslebens charakterisiert.

Zam Verständnis der dritten Plase, die die Hochflut der philosophisehen 'Tätigkeit bedeutet, ist rorauszuschicken, daß der Begrifl der "Weiterbildung" in engere Grenzen zu fassen ist, als die sind, die er in der Geschichte der modernen Philesophie besitzt. Venn die P'rinzipien einerseits und die Summe der Erfahrungstatsachen andererseits dio gleichen sind, damn kömen verschicdeno Systeme nur in sekundären Punkten varijeren. Dies ist für den breiten Strom der nachgazalischen l'hilosophie der Fall. Das empirische Material k 0 n $u$ t e mur das naive. Woltbild der alltäglichen Erfahrung, nicht das nach modener Methode mit Teleskop und Mikroskop erschlossene sein. Ferner sind die allgemeinen Denkprinzipien- und die Voraussetzungen des naiven, steflenweise zighlaft britischen ('Túsi 1273 f) Realismus die gleichen. Daher müssen die großon Züge der Systeme dieselben bleiben, und eine Weiterbildung ist nur i n nerhall dieser groben Linien möglich. Sio nimmt die Gestalt des Ausbanes im kleinen und der (sclseinbaren) Vertiefung dor Begrille durch genanere und mohr ins rinzehe gehende. Distinktionen an und verhält sich zu den äteren Werken, wie sich \%. B. der Kommentar Cajetans zur Summa theologiea des Thomas von Aquin verhailt. Un so molir sind bei dieser sachlage die sporadisch mol flüchtig auftretenden antirenlistischeol Bestreloungen (siehe Gazáli) ins duge zu fassen. Ibo Chaldún $1406+$ stellte den Grundsat\% anf, die Wissenschaft. besonders die der Geschichte, kömne nur die GesetzmäBigkeit der Erscheinungen foststellen. Von einer nicht der biffahrung zugänghlichen Welt könnten wir nichts wissen.

Unbeirrt durch solehe Kritilion entfaltot sich mach Gavali eine fast mü̈bersehbar grobe philosophische Literatur. Ihn Sima latte die Jogik in Werken dargestellt, die für den alltäglichen Gebranch zu umfangreich waren. Daher muBten klare und kurze liompendien geschaffen werden. Die bekanntesten stammen von Zaunúgi $1203+$ (Encheiridion studiosi), Salkkíki $1229+$ (Der Sehlüsse] zu den 
Wissenschaften), Hawingi $1248+$ Abhari $1264+$ (Die Führung zur Weisheit und die Isagroge), Kátibi $1276+$ (Handbuch der Logik für den liürsten Schemseddín usw.), Urmawi 1283 † (Aufgangsorte der Jichter), Násafi $1288+$, Samarkándi $1291+$ (Dethodik), Taftazáni $1389+$, Ahdari 1534t (Die Leiter der Logik). Die Geschichte dieser Handhïcher ist fast identisch mit der Geschichte der Logik im Islam. so ïberaus zahlreich sind ihre Kommentare und die Glossen und Superglossen zu diesen Kommentaren. Man darf über dieselben nicht kurzweg den Stah brechen, aus dem einfachen Grunde, weil sie die Form von Kommentaren und Glossen laben. Diss wäre eine Beurteilung des Inhaltes nach der zuläligen ä ub cren fiestalt d e r D a r s te ll $\| \mathrm{ng}$. In dieser damals ïblichen Darstellungsart worden häufig gan\% origrinelle, selbständige (iedanken entwickelt und das zu kommentierende Werk weiter ausgebaut oder sogar bekämpft.

An das P'roblem der spekulativen Theologic des Islam treten die (ieister mit aristotclischer Schulung heran. Násafis $1142+$ „Dogmen" bilden ein gesuchtes (Ohjekt philosophischer Lommentatoren. Gleiches gilt von Uschi $1173 \uparrow$. Schirázi $1310 \nmid$ und Gámi $1492 \dagger$ hetätigen sich als Mystiker, lyi 1335 f als Logiker und spekulativer Theologe, Rázi (at-tahtáni) $1336+$ t als Logiker, Dauwâni lóol † (Gottesbeweis)

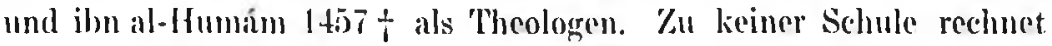
sich ibn Taimíja 13eg †. Hagzáde 1488 † kritisierte Gazális „Vernichtungr der Philosophen"n; Fanári 1431 †, Kiúschgi 1474 f. Sanúsi 1486 †, ibn Zarrák 1493 † sind bekannt als Mystiker. Kamál Páscha

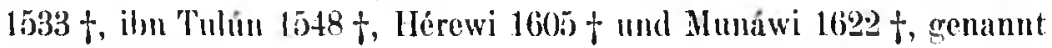
die Krone der Mystiker, sind Enzyklopädisten, wie anch Náblusi $1731+$ und Bekri $1749 \%$. Philosophische Enzyklopädien finden sich sehr häufig. Sie wollen das Gesamtwissen der Zeit darstellen und beginnen mit der Logrik als dem Weg zur Wissenschaft, stellen dann In der Physik das zeitgenössische Bild rom Weltall dar und endigen in der Metaphysik mit Jehren über cine deduktiv orschlossene, unsichtbare Welt und über die Prinzipien der Einzelwissenschaften. Vielfach nehmen diese Enzyklopädien die Gestalt von terminologischen, Würterbüchern an, so bei Hwárimi 997 †. Kascháni $1330 \dagger$, Nuwáiri 1332 †, Mahbúbi 1346 †, $\Lambda$ kfáni 1348 †, Corgáni 1413 †, IBistámi $1454+$. Taftazani (Haf́d) 1500 †, Sujúti $1505 \%$, den vielleicht fruchtbarsten Schriftsteller aller Zeiten und Zonen, Taschköprizáde 1560 †, Sacalilyzáde 1737 t, Farúki $1745+$ usw. Dic erste, die aristo- 
1elische Form wurde von vielen der bereits genannten Philosophen bevorzugt, z. B. ron ibn Sína $1037+$, Suhrawárdi $1191+$, Abhari J20 $6+$ †, Kátibi 1276 †, Schahrazuri un 1270 und Schirázi 1640 (Sidredelín).

Eine grobe Anzahl von liürstenspiegoln zeigen uns die pädagogisehen, ethischen und politischen Grundsätze der verschiedenen Epochen. Am bekanntesten sind Gazáli 1111 †, Tortúschi $1126 \%$ Adawi 125 5 †. Jurch Schriften rein ethischen Inhaltes sind besonders bekannt geworden ibn Maskawáil 1030 †, Gíli $1365 \dagger$ (,Der vollkommene Mensch"), Scharáni $1565 \%$ Die gesamte Rechtswissenschaft ist ferner in der ,Geschichte der Ethik im Islan", itso an dieser Stelle, nach ihren allgemein-ethischen Grundsätzen zo rewähnen. Desgleichen die Theologie und Mystik. Als praktische Philosophie, der theoretischen gegenüberstehend, behandeln anch die Philosophen die ethischen liagen im Anhang der Motaphysik.

Grob ist ferner die Awzahl der spekulativ interessierten Schriftstoller des vergangenen und jetzigen .Jahrhunderts. . Gurgi Zaidán, in Igypten lebend, hat dieselben in oinem besonderen llerke biographisch behandelt (Kairo 19(1)-1903). Die Produktion tritt häufigr in form von Kommentaren zu älteren Werken auf, vielfach nimmt sice anch selbständige Gestalt an. Die Färbung ist durchschnittlich theologisch- und mystisch-spekulativ oder logisch. Interessant ist es, zo sehen, welche Philosophen Farugi im 18. Jahrhmondert besonders schätzt. Neben den genannten und bekamnteren sind es llindi 1562 † (..Die fünl Substanzen"), Mäibudi 1475) ( Kommentar zu Abharis ..Die Fï̈hrung zur Weisheit"), Herewi 160) † (Glosse zum liommentar Corgánis zu den stationen des Igi), ilon Fúrac al Isbaháni 1015 t (Definitionen der Rechtsprinzipien), Isfaháni 1348 f (Kommentar der I)ogmatili von Tusi), (Yuwaini 1085, der Lehrer Gazális (,,Die Blätter"6), Bakiláni 1012 †, Salikúti 1656 † (Glossen), Hajáli 1456 † (Clossen), Ardabili 1543\% (Glossen), Nawawi $1278 \%$ Amidi $1233 \%$, Baidawi leg6† (,Die Aufgänge der Lichter", Mctaphysik), Lári 1506 † (Glossen zu Gámi 1492 t), Margináni 1197t, Tibrra 1494f (Kommentar zu Igi 1355) f) usw. Besonders häufig werden Kommentare, Glossen und Superglossen zitiert, seltener die glossierten und kommentierten Originale, dem in ersteren sind die Cedanken schärfer distinguiert und weiter ausgesponnen. Es scheint vielfach, daß sie fïr Farúqi sogar gr ö b e re $n$ Wert liaben als die glossierten Ichrbücher. 
Zum Schlusse sei erwähnt, dab auch das Problem der Geschichte der Philosophie sehr häufig, hesonders von theologischer und mystischer Seite behandelt wurde, so von Rázi 1209\%, Scharáni 155\% (Mystiker), ibn al-Múrtada 1437 (Jurist), Záhiri (ibn Mazm) 1064 † und Schahrastáni $1153+$ (Theologe), Nuhbbi $1699 \dagger-$ gamz ab)gesehen von den dureh ihre biographisehen Riesenwerke bekannten: Ishák an-Nadím 995 †, Kifti 1248 †, ibn abi Usaibia 1270 \%, Náwawi $1278+$, ibn Hallikán $1282 \uparrow$, Hággi Halsfia $1658 \uparrow$.

Bis jetzt sind ungefähr tausend Philosophen aus dem Islam, wenigstens dem Namen nach, bekannt. Bei grïndlicherer Ausbeutung der philosophischen und biographischen literatur wird sich diese bahl sicherlich noch um einige. Hunderte vermehren lassen. Die Masse der philosophischen literntur ist eine auberordentlich grobse. K/u ilı sind nicht nur die unter philosophischem Titel stehenden Werke zu rechuen, sondern anch manche andere, \%. 13. viele Korankoinmentare. Farúci zitiert solche geradezu als philosophische Werke; denu es ist unzweifelhaft und psychologisch notwendig, daß die Mystikel ihre indisch-neuplatonischen Gedanken, die Philosophen aristotelische Vorstellungen im lioran wiederfinden. Alle Stichproben lieforn immer wieder neue Bestätigungen dieser Vermutung.

Die Philosophie cines Volkes ist cin Schanuspiel, das sich innerhalb dor Szenerio der gesamten Geisteskultur des betreffenden Volkes abspielt, zumal wenn das Bildungsinteresse, besonders das der Philosophen, nicht cin spezialwissenschafuliches, sondern ein allgemeines ist. Zur Charalkterisicrung der islamischem Geisteslunlfur ist daher zu betonen, dab der Islam auf fast allen Gebieten der lï̈nste und Wissenschaften durchaus beachtenswerte locistungen aulouweisen lat, die jedenfalls in den Naturwissenschaften (Physik, Astronomir, Astrologie, Alchimie, (hemie, Zoologie, Botanik, Mineralogrie, Medizin) dor Geschichte, Geographir, Sprachwissenschaft und Rechatswissenschaft die gleichzoitige Kultur Europas bis 1000) sehr in Schatten stellen. Zu bewundem ist vor allem die grobe /ähigkeit, mit der der

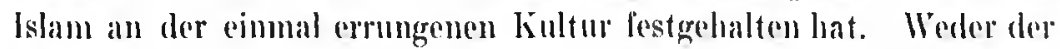
Mongolensturm noch die Türkenherrsehaft haben sie vernichten kömnen. Sogar in den schlimmsten Zeiten war das Interesse an der Philosophie und die schöpferische Tätigkeit aul diesem (iebiete immer rege.

Diese kurze Skizzo verfolgt die Absicht, die groben Gebiete anzudeuten, die der Forschung noch zu erschlieben sind, und das Interesse 
an der Geschichte der philosophischen Ideen im Islam zu orwedken. Eine Gesamtdarstellung dieses Gegenstandes setzt eine grobe Anzahl von Vorarbeiten voraus, die noch der Lösung harren. So viele Namen von Philosophen im Islam - so viele Probleme. Von hesonderer Wichtigkeit wäre die Gesehichte der Akademien, deren es eine grobe Zahl gegeben hat. Diese Vorarbeiten werden dadurch erleichtert, lab der Orient seit mehreren Jahren bestrebt ist, die Originalwerke vergangener Jahrhunderte in genïgend guten Publikationen \%ugainglich zu machen. Diese Taitigkeit wird, so steht zu erwarten, durch die im Oktober 1908 in Kairo eröfnete Universitiit, die nach enropiiischem Muster eingerichtet ist, einen neuen Aufschwung erleben. Mögen die dortigen Bestrebungen auch in Furopa ihren Widerhall finden in einem gesteigerten Interesse an dem Kampfe des Jslam um den Fortschritt der Finltur. 